\title{
Defeating the Hydra of Excess Cardiovascular Disease Risk in Rheumatoid Arthritis - How Close Are We to Completing the Task?
}

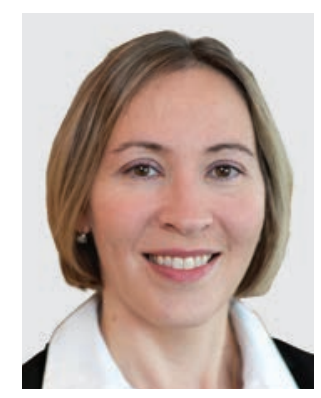

\author{
Elena Myasoedova ${ }^{1}$ (D)
}

For his Second Labor, Heracles was tasked with slaying the Hydra, a serpentine monster from ancient Greek mythology who possessed many heads. When Heracles attempted to decapitate the monster, two new heads grew in place of each severed head, an expression of hopelessness of such struggle for anyone but the hero. The invulnerability of the Hydra was because of its one immortal head. Once the immortal head was cut off, the monster was defeated, and Heracles' second task was completed.

Much like slaying the monstrous Hydra, conquering the disproportionally increased risk of nearly all forms of fatal and nonfatal cardiovascular disease (CVD) in patients with rheumatoid arthritis (RA) compared to the general population has been an unsolved lingering problem, long awaiting the arrival of its hero. Fueled by systemic autoimmune inflammation, this 1.5 - to 2 -fold excess CVD risk has been invulnerable to traditional CVD management strategies. Growing attention to tight control of RA disease activity and widespread use of conventional synthetic disease-modifying antirheumatic drugs (csDMARD) and biologic DMARD (bDMARD) have dramatically changed the landscape of RA outcomes in the new millennium, providing hopes for defeating the increased CVD risk.

A metaanalysis of observational studies highlighted the potential for up to 30\% CVD risk reduction in RA with the use of methotrexate (MTX) and tumor necrosis factor- $\alpha$ inhibitors $(\mathrm{TNFi})^{1}$. A large retrospective study from Sweden demonstrated even greater benefits, including the reduction of CVD risk in RA patients who responded to TNFi to that of the general popula-

Dr. Myasoedova is supported by the Louis V. Gerstner, Jr. Fund at Vanguard Charitable.

${ }^{I}$ E. Myasoedova, MD, PhD, Associate Professor of Internal Medicine, Senior Associate Consultant, Division of Rheumatology, Department of Internal Medicine, Mayo Clinic, Rochester, Minnesota, USA.

Address correspondence to Dr. E. Myasoedova, Mayo Clinic College of Medicine and Science, Division of Rheumatology, 200 1st St. SW, Rochester, MN 55905, USA. Email: myasoedova.elena@mayo.edu. tion level ${ }^{2}$. Remission of RA disease activity, especially if achieved early in the disease course, has been associated with reduction of the CVD risk to a level comparable to the general population level ${ }^{3,4}$, identifying systemic inflammation as the "immortal head" of the excess CVD risk in RA, and reiterating the importance of early and effective control of RA disease activity. Research on comparative effectiveness of various antirheumatic treatments and combinations on clinically relevant ("hard") CVD outcomes in real-world population is gaining momentum.

In this issue of The Journal, Ozen, et al examine comparative effects of TNFi, non-TNFi biologics, and tofacitinib versus csDMARD on rates of nonfatal and fatal CVD events in adult patients with RA ( $\mathrm{n}=18,754)$ participating in FORWARD, the National Databank for Rheumatic Diseases longitudinal prospective observational study, between 1998 and 2017. The authors can be complimented on the rigorous methodological approach aimed at minimizing bias and confounding inherent to observational studies. They carefully address the possibility of overlap or carryover effect from the previous treatments by allowing a 3-month risk window for exposure lag following discontinuation of the previous medications, adjustment for the number of prior csDMARD and bDMARD, and use of mutually exclusive medication categories with time-varying exposure variables. Although not employing propensity scoring, given the multiple potential confounders, the authors used a comprehensive list of adjustors, including sociodemographic factors, traditional CVD risk factors and comorbidities, use of medications influencing CVD risk, RA disease characteristics, prior antirheumatic medication use, prednisone exposure, and calendar year. The study features several important findings.

Reduction in risk of CVD events with TNFi use

Ozen, et a ${ }^{5}$ report an $18 \%$ reduction in risk of composite CVD events [i.e., myocardial infarction (MI), stroke, hospitalized heart failure (HF), death from CVD] with TNFi use versus any csDMARD. When patients with prior CVD were excluded, 
the association remained statistically significant, with $17 \%$ risk reduction in incident CVD with TNFi use versus csDMARD.

These findings extend the existing knowledge of the role of TNFi in primary and secondary CVD prevention in $\mathrm{RA}^{6}$. Significant CVD risk reduction was observed for the individual CVD events (i.e., MI, stroke, CVD-related death) with no increase in risk of hospitalized HF with TNFi use. When the csDMARD comparator group was prespecified to include MTX, TNFi use was associated with reduced risk of MI, while association with other individual outcomes or composite CVD outcome did not reach statistical significance. In this context, there are 2 questions to ask: To what extent may bDMARD, including TNFi as the most widely used bDMARD, be superior to MTX for CVD risk reduction, and what are the underlying mechanisms of this additional CVD benefit?

CVD benefits of TNFi use on surrogate and "hard" CVD outcomes in RA are becoming increasingly established and presumed to be largely due to effective control of systemic inflammation as observed in TNFi responders, current but not prior TNFi users, and those with prolonged TNFi use $\mathrm{e}^{2,7,8,9,10}$. The extent of TNFi-specific effects on CVD health beyond inflammation control is an area of active research. Studies of surrogate CVD outcomes show plaque stabilization, reduction in aortic stiffness, and improvement of endothelial function in TNFi users with RA, irrespective of RA disease activity ${ }^{8,11,12}$. Whether these improvements in surrogate CVD markers translate into improved long-term CVD outcomes that may account for superior CVD benefits in TNFi users, and whether this is a class effect, requires further study. Recent preliminary data from the Norwegian DMARD (NOR-DMARD) registry using data from 3251 patients with RA show higher risk estimates for a composite CVD outcome in RA remission on csDMARD (HR 0.75) compared to patients on various bDMARD $(\text { HR } 0.3)^{13}$. While the difference did not reach statistical significance, these findings substantiate the rationale for further studies evaluating effects of different modes of inflammation control on CVD risk in RA.

\section{CVD benefits of non-TNFi use}

As one of the main findings, Ozen, et at report a $50 \%$ risk reduction in CVD events (primarily MI) with abatacept (ABA), a selective inhibitor of $\mathrm{T}$ cell co-stimulation, as compared to csDMARD. A subgroup analysis that excluded patients with prior TNFi use revealed a similar degree of CVD risk reduction of $~ 50 \%$ with $\mathrm{ABA}$ versus csDMARD, although the strength of association was attenuated. Similar to TNFi use, this CVD risk reduction with $\mathrm{ABA}$ was primarily in comparison to non-MTX csDMARD and became borderline significant when the reference group was set to include MTX (rather than any csDMARD).

While the role of ABA in CVD is less studied as compared to $\mathrm{TNFi}$, the findings of Ozen, et al ${ }^{5}$ concur with the growing body of research suggesting clinical CVD benefits of ABA as a first-line and second-line non-TNFi biologic ${ }^{14,15,16}$. Individual observational studies suggest superior CVD benefits of $A B A$ as compared to TNFi in subgroups of patients with RA who have high CVD risk. Indeed, decrease in risk of CVD outcomes (primarily MI) with $\mathrm{ABA}$ was somewhat more prominent than with TNFi use in large US-based cohorts of patients with RA of advanced age, or who have preexisting CVD and diabetes mellitus ${ }^{14,15,17}$. However, a recent metaanalysis of large cohort studies showed no difference in risk of major adverse cardiac events between ABA and TNFi in patients with RA overall, without examination of CVD risk subgroup ${ }^{18}$. Similarly, Ozen, et at found no additional CVD benefit of ABA compared to TNFi use in RA. Randomized controlled studies (RCT) addressing CVD safety of $A B A$ in patients with RA overall and across different baseline CVD risk phenotypes would be helpful in informing treatment decisions about the use of $\mathrm{ABA}$ in RA.

The study by Ozen, et a ${ }^{5}$ did not detect any statistically significant associations with regard to CVD risk for other non-TNFi biologics and tofacitinib compared to csDMARD, although event numbers were small. Previous observational studies and clinical trial data suggest a good potential for interleukin 6 inhibitors in management of CVD risk, particularly $\mathrm{MI}$ risk, in RA and acceptable comparative CVD safety of tocilizumab versus $\mathrm{TNFi}^{18,19}$. Janus kinase (JAK) inhibitors demonstrate higher proportion of patient responders according to the American College of Rheumatology 20/50/70 response rates than other targeted therapies used in RA, suggesting that more patients with RA may be benefiting from JAK inhibitor use from a standpoint of inflammation control. However, information on comparative CVD safety of these compounds is scarce ${ }^{18}$, and there is a possibility of increased risk of venous thromboembolism with JAK inhibitors ${ }^{20}$. More studies with guards against selection bias and confounding are needed to evaluate effect of JAK inhibitors on CVD risk.

\section{Role of glucocorticoid use in combination with DMARD on CVD outcomes}

Adverse CVD effects of systemic glucocorticoid (GC) use are well established ${ }^{10,21}$. Ozen, et a ${ }^{5}$ used a weighted cumulative exposure (WCE) model that considered the effects of dosage, duration, and timing of GC use on the risk of CVD in RA. Augmenting the existing evidence of dose- and duration-dependent increased risk of CVD events with GC use, this study found that GC exposure as WCE of prednisone was associated with increased CVD risk in RA (HR 1.15, 95\% CI 1.11-1.19). Importantly, concomitant use of GC with DMARD in this study abated the protective effect of TNFi, ABA, and MTX. While confounding by indication is a possibility (i.e., patients with more severe RA and inherent adverse CVD risk profile may be more likely to use GC), their adverse cardiometabolic effects are well known and may play a role in outweighing the CVD benefits of csDMARD and bDMARD use. This reinforces the recommendations for minimal possible GC exposure as one of the overarching principles of CVD management in patients with $\mathrm{RA}^{22}$.

As the evidence on association between the use of antirheumatic medications and CVD outcomes in patients with RA is mounting, it is important to understand the implications of these findings in the context of existing literature on trends in CVD in RA. Has there been a change in epidemiology of CVD 
in RA in recent decades? What is the effect on the population of csDMARD and targeted therapies on CVD morbidity and mortality in RA?

Following decades of persistent excess in risk of CVD in RA, recent population-based studies from several developed countries show a closing of the CVD mortality gap and diminishing gap in excess incidence of CVD in RA versus the general population ${ }^{23,24}$. While causation cannot be established from the observational studies, these improvements in CVD outcomes in RA come in parallel with widespread use and early initiation of MTX, growing use of targeted therapies, and declining disease activity of RA at baseline and up to 10 years of follow-up ${ }^{25}$. These trends in CVD incidence and mortality were observed primarily in high-income countries and are unlikely to be universal, but they highlight a potential for significant improvements in CVD outcomes in the setting of improved control of RA disease activity with csDMARD and bDMARD. Prospective studies, ideally RCT, are needed to define the most effective approaches for primary and secondary CVD prevention in RA.

In summary, heroic efforts of the rheumatology community have led to increasing recognition of early initiation of DMARD, acceptance of a "treat-to-target" (i.e., treat-to-remission) approach, and discovery of an expanding range of targeted therapies as means of improving outcomes of RA disease overall, with growing potential for improved CVD outcomes in RA. Successfully combating systemic inflammation as the Hydra's immortal head makes decreasing CVD risk in RA an achievable goal in patients with access to effective antirheumatic treatments.

\section{REFERENCES}

1. Roubille C, Richer V, Starnino T, McCourt C, McFarlane A, Fleming $\mathrm{P}$, et al. The effects of tumour necrosis factor inhibitors, methotrexate, non-steroidal anti-inflammatory drugs and corticosteroids on cardiovascular events in rheumatoid arthritis, psoriasis and psoriatic arthritis: a systematic review and meta-analysis. Ann Rheum Dis 2015;74:480-9.

2. Ljung L, Rantapaa-Dahlqvist S, Jacobsson LT, Askling J. Response to biological treatment and subsequent risk of coronary events in rheumatoid arthritis. Ann Rheum Dis 2016;75:2087-94.

3. Myasoedova E, Chandran A, Ilhan B, Major BT, Michet CJ, Matteson EL, et al. The role of rheumatoid arthritis (RA) flare and cumulative burden of RA severity in the risk of cardiovascular disease. Ann Rheum Dis 2016;75:560-5.

4. Arts EE, Fransen J, Den Broeder AA, van Riel P, Popa CD. Low disease activity (DAS28 $\leq 3.2$ ) reduces the risk of first cardiovascular event in rheumatoid arthritis: a time-dependent Cox regression analysis in a large cohort study. Ann Rheum Dis 2017;76:1693-9.

5. Ozen G, Pedro S, Michaud K. The risk of cardiovascular events associated with disease-modifying antirheumatic drugs in rheumatoid arthritis. J Rheumatol 2021:48:648-55.

6. de La Forest Divonne M, Gottenberg JE, Salliot C. Safety of biologic DMARDs in RA patients in real life: a systematic literature review and meta-analyses of biologic registers. Joint Bone Spine 2017;84:133-40.

7. Lee JL, Sinnathurai P, Buchbinder R, Hill C, Lassere M, March L. Biologics and cardiovascular events in inflammatory arthritis: a prospective national cohort study. Arthritis Res Ther 2018;20:171.

8. Karpouzas GA, Ormseth SR, Hernandez E, Budoff MJ. Biologics may prevent cardiovascular events in rheumatoid arthritis by inhibiting coronary plaque formation and stabilizing high-risk lesions. Arthritis Rheumatol 2020 Apr 21 (E-pub ahead of print).

9. Dixon WG, Watson KD, Lunt M, Hyrich KL, British Society for Rheumatology Biologics Register Control Centre C, Silman AJ, et al; British Society for Rheumatology Biologics Register. Reduction in the incidence of myocardial infarction in patients with rheumatoid arthritis who respond to anti-tumor necrosis factor alpha therapy: results from the British Society for Rheumatology Biologics Register. Arthritis Rheum 2007;56:2905-12.

10. Nurmohamed M, Bao Y, Signorovitch J, Trahey A, Mulani P, Furst DE. Longer durations of antitumour necrosis factor treatment are associated with reduced risk of cardiovascular events in patients with rheumatoid arthritis. RMD Open 2015;1:e000080.

11. Deyab G, Hokstad I, Whist JE, Smastuen MC, Agewall S, Lyberg $\mathrm{T}$, et al. Methotrexate and anti-tumor necrosis factor treatment improves endothelial function in patients with inflammatory arthritis. Arthritis Res Ther 2017;19:232.

12. Vlachopoulos C, Gravos A, Georgiopoulos G, Terentes-Printzios D, Ioakeimidis N, Vassilopoulos D, et al. The effect of TNF- $\alpha$ antagonists on aortic stiffness and wave reflections: a meta-analysis. Clin Rheumatol 2018;37:515-26.

13. Berg IJ, Lillegraven S, Kristianslud E, Kvien TK, Provan SA. Not all the same? Reaching remission reduces the risk of CVD in patients with RA, but patient on biologics may be better protected [abstract]. Ann Rheum Dis 2020; Suppl 79.

14. Jin Y, Kang EH, Brill G, Desai RJ, Kim SC. Cardiovascular (CV) risk after initiation of abatacept versus TNF inhibitors in rheumatoid arthritis patients with and without baseline CV disease. J Rheumatol 2018;45:1240-8.

15. Kang EH, Jin Y, Brill G, Lewey J, Patorno E, Desai RJ, et al. Comparative cardiovascular risk of abatacept and tumor necrosis factor inhibitors in patients with rheumatoid arthritis with and without diabetes mellitus: a multidatabase cohort study. J Am Heart Assoc 2018;7:e007393.

16. Hsieh MJ, Lee CH, Tsai ML, Kao CF, Lan WC, Huang YT, et al. Biologic agents reduce cardiovascular events in rheumatoid arthritis not responsive to tumour necrosis factor inhibitors: a national cohort study. Can J Cardiol 2020 Jan 15 (in press).

17. Zhang J, Xie F, Yun H, Chen L, Muntner P, Levitan EB, et al. Comparative effects of biologics on cardiovascular risk among older patients with rheumatoid arthritis. Ann Rheum Dis 2016; 75:1813-8.

18. Singh S, Fumery M, Singh AG, Singh N, Prokop LJ, Dulai PS, et al. Comparative risk of cardiovascular events with biologic and synthetic disease-modifying antirheumatic drugs in patients with rheumatoid arthritis: a systematic review and meta-analysis. Arthritis Care Res 2020;72:561-76.

19. Giles JT, Sattar N, Gabriel S, Ridker PM, Gay S, Warne C, et al. Cardiovascular safety of tocilizumab versus etanercept in rheumatoid arthritis: a randomized controlled trial. Arthritis Rheumatol 2020;72:31-40.

20. Sepriano A, Kerschbaumer A, Smolen JS, van der Heijde D, Dougados M, van Vollenhoven R, et al. Safety of synthetic and biological DMARDs: a systematic literature review informing the 2019 update of the EULAR recommendations for the management of rheumatoid arthritis. Ann Rheum Dis 2020;79:760-70.

21. Wei L, MacDonald TM, Walker BR. Taking glucocorticoids by prescription is associated with subsequent cardiovascular disease. Ann Intern Med 2004;141:764-70.

22. Agca R, Heslinga SC, Rollefstad S, Heslinga M, McInnes IB, Peters $\mathrm{MJ}$, et al. EULAR recommendations for cardiovascular disease risk management in patients with rheumatoid arthritis and other forms of inflammatory joint disorders: 2015/2016 update. Ann Rheum Dis 2017;76:17-28.

23. Myasoedova E, Gabriel SE, Matteson EL, Davis JM, 3rd, Therneau 
TM, Crowson CS. Decreased cardiovascular mortality in patients with incident rheumatoid arthritis (RA) in recent years: dawn of a new era in cardiovascular disease in RA? J Rheumatol 2017; 44:732-9.

24. Holmqvist M, Ljung L, Askling J. Acute coronary syndrome in new-onset rheumatoid arthritis: a population-based nationwide cohort study of time trends in risks and excess risks. Ann Rheum Dis 2017;76:1642-7.
25. Gwinnutt JM, Symmons DPM, MacGregor AJ, Chipping JR, Marshall T, Lunt M, et al. Have the 10-year outcomes of patients with early inflammatory arthritis improved in the new millennium compared with the decade before? Results from the Norfolk Arthritis Register. Ann Rheum Dis 2018;77:848-54. 